\title{
Health Professional Burnout: An (Un)Ethical Consequence of Modern Health Care?
}

\author{
Michael Shevell
}

Keywords: Ethics, Professionalism, Health systems

doi:10.1017/cjn.2019.280

Can J Neurol Sci. 2019; 46: 682-683

Burnout, defined as a state of physical, emotional and motivational exhaustion that is the result of protracted stress or frustration, has become an increasing issue of concern in Canada's health system, yet its ethical dimensions have largely been ignored. Subjectively experienced as feeling exhausted, fatigued, or simply overwhelmed by tasks, it leads to depersonalization, cynicism and a decline in motivation and vigilance. The net result for the physician is an increasing disengagement from his or her professional, vocational and organizational obligations. Cognitively, the affected professional experiences a decline in attention, memory and executive skills. Ultimately, there is a loss of compassion, empathy, esteem, confidence, meaning and professionalism. The resulting negative effects on the actual quality of care provided, patient safety and access to care have been documented. ${ }^{1}$ Burnout is estimated to affect 30-60\% of health care providers, with $60 \%$ of American neurologists admitting to having experienced at least one symptom of burnout. $^{2}$ As an academic leader (chair of a university department and pediatrician-in-chief of a tertiary children's hospital numbering more than 200 specialty and subspecialty physicians), faculty wellness and work-life integration has become an increasing personal preoccupation to ensure our mandates of academic excellence and service provision.

In health care professions, traditionally, burnout has been attributed to an ever-increasing pace of change, at times verging on perceived chaos to the front-line clinician, together with an ever-escalating time pressure typically conceptualized as providing care for a greater volume of ill patients. ${ }^{2}$ This is combined with a lack of personal control or influence on work processes. Not coincidentally, these are linked to an increasing health care bureaucracy and a densely complex administrative structure, which is remote and distant, that has imposed a range of nonvalue-added, at least to the practicing professional, tasks.

Missing from the foregoing process-oriented analysis of the causes of burnout is an explicit consideration of the humane side of health care provision that depends on a cadre of highly motivated healthy physicians. This is the experience of "moral distress" by professionals. Moral distress is the product of the discrepancy between the belief in what is the right thing for one to do and external constraints imposed by an institution or a system that obliges the pursuit of limited courses of action. ${ }^{3}$ Simply stated, the ethically correct action does not necessarily equate with what one is required, or, more frequently, actually enabled, to do. At its extreme, when egregious, moral distress can lead to moral injury, wherein one engages reluctantly and with conscious awareness in an act of moral transgression against one's own deeply held and self-identifying core values and principles. ${ }^{3}$ Doing so constitutes a breach of or rupture in one's integrated constructed sense of self and humanity. Such an occurrence can lead to profound emotional shame and a breakage of one's world view. This is analogous to the trauma experienced in post-traumatic stress disorder.

The increasing number of pressures and constraints on health care professionals places these highly qualified and difficult-toreplace individuals at high risk for moral distress and injury. The health professions are meant as "callings", not "jobs." For health care professionals, long periods of demanding training often entail personal sacrifices compared to peers who have chosen non-health career paths. The health professions aspire to, and ingrain in their members, high standards of professionalism that are frequently altruistic and, at times, contextually subject to nonattainment. Rituals (e.g. white coat ceremonies) reinforce one's membership in a rarefied group. Unfortunately, due to external constraints, all-too-often high-quality healing and health care remains an aspirational good for professionals who have incorporated into their world view a range of noble aims attached to their profession. For many, beyond fatigue and exhaustion (something they are used to through training), it is the experience of moral distress and injury that may be the proverbial "straw that breaks the camel's back," leading to burnout.

Morality in any sphere is tied to ethics rooted in consensual responsibilities and obligations to others. Thus, to be effective, a system-wide response to burnout should be driven by ethics and moral imperative. Obligations are reciprocal, owed to both patients and care providers. Primum non nocere also applies to the provider if a sustainable model of quality health care delivery is the intended goal for our society. The provider's vital role in health care and his or her professional autonomy, expertise and

From the Departments of Pediatrics and Neurology/Neurosurgery, McGill University, Montreal, Quebec, Canada; Montreal Children's Hospital-McGill University Health Centre, Montreal, Quebec, Canada

Received May 21, 2019. Final Revisions Submitted August 4, 2019. Date of Acceptance August 8, 2019.

Correspondence to: Michael Shevell, Pediatrician-in-Chief, McGill University Health Centre, 1001 Decarie Blvd, Montreal, QC H4A 3J1, Canada. Email: michael.shevell@muhc.mcgill.ca 
priorities must be respected and reinforced. ${ }^{4}$ Top-down models of health system management that impose expectations for reasons of expediency and political gain that fail to take into account the moral imperatives of providers embodied in their innate professionalism and humanity will lead only to more moral distress and injury. Inevitably, more cracks will appear in the foundation, cracks that are the product of human casualties until the cumulative effects of these casualties result in a decline of the health care edifice.

The public has a vested moral interest in formulating a system that promotes the resilience of professionals and minimizes burnout. Maximizing access and sustainability, improving outcomes, and promoting the well-being of the population are the usual first principles that are used to justify public health policies and the structure of our health care systems. These moral goods as prime drivers rest substantially on an available and healthy professional workforce.

It is time that health care professionals, policy makers and the general public recognize the underlying moral dimensions of burnout. This recognition is an essential precondition to work collectively to articulate responses that minimize the distress and injury being experienced by our health care professionals.
Providing a moral framework to the structural analysis may also serve to expedite the formulation of remediating policies that have the broad support of all stakeholders, whose consensual apparent aim is a morally just, sustainable and effective health care system. Driven by an ethical imperative to minimize moral distress, health care professionals can broadly formulate solutions to minimize burnout and ensure the essential prerequisite of a healthy health care workforce.

\section{Disclosures}

The author has no conflicts of interest to declare.

\section{REFERENCES}

1. Shanafelt TD, Schein E, Minor LB, Trockel M, Schein P, Kirch D. Healing the professional culture of medicine. Mayo Clin Proc. 2019. Published Online July 11, 2019.

2. Sigsbee B, Bernat JL. Physician burnout: a neurologic crisis. Neurology. 2014;83:2302-06.

3. Fourie C. Moral distress and moral conflict in clinical ethics. Bioethics. 2015;29:91-7.

4. Epstein RP, Krasner MS. Physician resilience: what it means, why it matters, and how to promote it. Acad Med. 2013;88:301-03. 